SOI: $1.1 /$ TAS DOI: $10.15863 /$ TAS

\section{International Scientific Journal}

\section{Theoretical \& Applied Science}

p-ISSN: 2308-4944 (print) $\quad$ e-ISSN: 2409-0085 (online)

Year: 2015 Issue: 07 Volume: 27

Published: $30.07 .2015 \quad \underline{\text { http://T-Science.org }}$

SECTION 2. Applied mathematics. Mathematical modeling.
Liana Khusenovna Nazarova Postgraduate Student of the Differential Equations Department, Department of Mathematics, Kabardino-Balkarian State University, Nalchik, Kabardino-Balkaria, Russian Federation n.1.h@yandex.ru

Vadim Nikolayevich Lesev Candidate of Physical and Mathematical Science, Associate Professor, Head of the Differential Equations Department, Department of Mathematics, Kabardino-Balkarian State University, Nalchik, Kabardino-Balkaria, Russian Federation

\title{
ABOUT TWO BOUNDARY VALUE PROBLEMS FOR EVEN-ORDER NONLINEAR EQUATIONS
}

Abstract: The paper researches two boundary value problems for second-order and fourth-order nonlinear equations. Existence of regular solution of the fourth-order equation problem is proved by the quasi-stationary method. The boundary value problem for the fourth-order equation is researched by the variable separation method.

Key words: nonlinear equation, partial differential equation, variable separation method, quasi-stationary method.

Language: Russian

Citation: Nazarova LK, Lesev VN (2015) ABOUT TWO BOUNDARY VALUE PROBLEMS FOR EVENORDER NONLINEAR EQUATIONS. ISJ Theoretical \& Applied Science 07 (27): 118-121.

Soi: http://s-o-i.org/1.1/TAS-07-27-20 Doi: crossef http://dx.doi.org/10.15863/TAS.2015.07.27.20

\section{О ДВУХ КРАЕВЫХ ЗАДАЧАХ ДЛЯ НЕЛИНЕЙНЫХ УРАВНЕНИЙ ЧЕТНОГО ПОРЯДКА}

Аннотация: В работе исследованы две краевые задачи для нелинейных уравнений второго и четвертого порядков. Доказательство существования регулярного решения задачи для уравнения второго порядка проведено квазистационарным методом. Краевая задача для уравнения четвертого порядка исследована методом разделения переменных.

Ключевые слова: нелинейное уравнение, уравнение в частных производных, метод разделения переменных, квазистационарный метод.

\section{1. Введение}

Теория краевых задач для нелинейных уравнений в частных производных относится к практически значимым разделам дифференциальных уравнений. Подобные задачи достаточно часто становятся основой математических моделей, применяемых в биологии, физике и других науках [1-8].

В настоящей работе исследованы две краевые задачи для нелинейных уравнений в частных производных второго и четвертого порядков. Вопрос существования регулярного решения в случае уравнения второго порядка исследован на основе квазистационарного метода, а доказательство существования решения для уравнения четвертого порядка проведено методом разделения переменных.
1. Краевая задача для нелинейного уравнения 2-го порядка

Рассмотрим краевую задачу для нелинейного уравнения 2-го порядка

$$
\frac{\partial U}{\partial t}=\frac{\partial}{\partial x}\left(U \frac{\partial U}{\partial x}\right)+a U,
$$

где $a=$ const, с условиями:

$$
\begin{gathered}
U_{x=0}=\left.U\right|_{x=l}=\alpha(t), \\
\left.U\right|_{t=0}=\gamma(x) .
\end{gathered}
$$

в области $\Omega=\{(x, t): 0<x<l, 0<t<l\}$.

Для исследования задачи (1) - (3) будем использовать квазистационарный метод, т.е. решение (1) уравнения будем искать в виде

$$
U(x, t)=u_{0}(x)+\varepsilon u_{1}(x, t),
$$

где $u_{0}(x)=\langle U(x, t)\rangle$ - усреднение по времени. 


\begin{tabular}{|c|c|c|c|c|c|}
\hline Impact Factor: & $\begin{array}{l}\text { ISRA (India) } \\
\text { ISI (Dubai, UAE } \\
\text { GIF (Australia) } \\
\text { JIF }\end{array}$ & $\begin{array}{l}=1.344 \\
=0.829 \\
=0.356 \\
=1.500\end{array}$ & $\begin{array}{l}\text { SIS (USA) } \\
\text { PИHЦ (Russia) } \\
\text { ESJI (KZ) } \\
\text { SJIF (Morocco) }\end{array}$ & $\begin{array}{l}=0.912 \\
=0.179 \\
=1.042 \\
=2.031\end{array}$ & ICV (Poland) \\
\hline
\end{tabular}

Из (1), относительно $u_{0}$, принимая во внимание (4), получим следующее обыкновенное дифференциальное уравнение

$$
u_{0} u_{0}^{\prime \prime}+u_{0}^{\prime 2}+a u_{0}=0 .
$$

С помощью замены $u_{0}^{\prime}=p, \quad u_{0}^{\prime \prime}=p p^{\prime}$, редуцируем уравнение (5) к виду:

$$
u_{0} p p^{\prime}+p^{2}=-a u_{0}
$$

Рассмотрим соответствующее однородное уравнение:

$$
u_{0} p p^{\prime}+p^{2}=0 .
$$

Разрешая (7), будем иметь:

$$
\frac{d p}{p}=-\frac{d u_{0}}{u_{0}} .
$$

Откуда, находим:

$$
p=\frac{c_{1}}{u_{0}} .
$$

Частное решение (8) будем искать методом вариации постоянных:

$$
p_{c}=\frac{c_{1}\left(u_{0}\right)}{u_{0}} .
$$

Подставляя (9) в (6), получим

$$
u_{0} \frac{c_{1}\left(u_{0}\right)}{u_{0}} \frac{c_{1}^{\prime}\left(u_{0}\right) u_{0}-c_{1}\left(u_{0}\right)}{u_{0}^{2}}+\frac{c_{1}^{2}\left(u_{0}\right)}{u_{0}^{2}}=-a u_{0} \text {. }
$$

Откуда, находим:

$$
\frac{c_{1}^{\prime}\left(u_{0}\right) c_{1}\left(u_{0}\right)}{u_{0}}=-a u_{0},
$$

или

$$
c_{1}\left(u_{0}\right)=\left(-\frac{2 a}{3} u_{0}^{3}\right)^{\frac{1}{2}} .
$$

Таким образом, частное решение (6) будет иметь вид:

$$
p_{c}=\left(-\frac{2 a}{3} u_{0}\right)^{\frac{1}{2}} .
$$

Производя обратную замену в (10), получим:

$$
u_{0}^{\prime}=\left(-\frac{2 a}{3} u_{0}\right)^{\frac{1}{2}} .
$$
иметь

Интегрируя полученное выражение, будем

$$
u_{0}(x)=-\frac{a}{6}(x+c)^{2},
$$

где $c=$ const .

Постоянную интегрирования $c$ определим позже.

Возвращаясь к исследуемому уравнению (1) и принимая во внимание (4), приходим к равенству [9]:

$$
\begin{aligned}
& \frac{\partial u_{1}}{\partial t}=\left(u_{0}+u_{1}\right) \frac{\partial^{2}\left(u_{0}+u_{1}\right)}{\partial x^{2}}+ \\
& +\left(\frac{\partial\left(u_{0}+u_{1}\right)}{\partial x}\right)^{2}+a\left(u_{0}+u_{1}\right)
\end{aligned}
$$

Входящую в (12) искомую функцию $u_{1}$ будем искать в виде экспоненциальной зависимости [10]:

$$
u_{1}(x, t)=A(x) e^{\omega t+\varphi_{0}} .
$$

Из (12) с учетом (11) и (13), в случае $A(x)=$ const определяем $\omega$ :

$$
\omega=\frac{2 a}{3} .
$$

Таким образом, окончательно для функции $u(x, t)$ можем записать:

$$
u(x, t)=-\frac{a}{6}(x+c)^{2}+A e^{\frac{2 a}{3} t+\varphi_{0}},
$$

где $\quad c, A, \varphi_{0}$ - произвольные числовые параметры.

Определим постоянные $\quad c, A, \varphi_{0}$ удовлетворяя (14) условиям (2) и (3). Действительно, будем иметь:

$$
\left\{\begin{array}{c}
\left.u\right|_{x=0}=-\frac{a}{6} c^{2}+A e^{\omega t+\varphi_{0}}=\alpha(t) \\
\left.u\right|_{x=l}=-\frac{a}{6}(l+c)^{2}+A e^{\omega t+\varphi_{0}}=\alpha(t) \\
\left.u\right|_{t=0}=-\frac{a}{6}(x+c)^{2}+A e^{\varphi_{0}}=\gamma(x)
\end{array}\right.
$$

Разрешая (15), находим:

$$
c=-\frac{l}{2} \text {. }
$$

Параметры $A, \varphi_{0}$ определяются в соответствии с типом популяционной модели.

Таким образом, решение задачи (1) - (3), представимо в виде (14).

\section{2. Краевая задача для нелинейного} уравнения 4-го порядка

В области $\Omega=\{(x, t): 0<x<l, 0<t<l\}$ рассмотрим уравнение

$$
U\left(U_{x x x x}+\alpha U_{x x}\right)-\beta U_{t}=0,
$$

где $\alpha, \beta=$ const $>0$.

Для уравнения (16) исследована задача.

Найти решение $U(x, t)$ в области $\Omega$, удовлетворяющее условиям:

$$
\begin{gathered}
U(0, t)=U_{x}(0, t)=U(l, t)=U_{x}(l, t)=0, \\
U(x, 0)=\tau(x),
\end{gathered}
$$

и условиям согласования $\tau(0)=\tau(l)=0$. 


\begin{tabular}{|c|c|c|c|c|c|c|}
\hline Impact Factor: & $\begin{array}{l}\text { ISRA (India) } \\
\text { ISI (Dubai, UAE } \\
\text { GIF (Australia) } \\
\text { JIF }\end{array}$ & $\begin{array}{l}=1.344 \\
=0.829 \\
=0.356 \\
=1.500\end{array}$ & $\begin{array}{l}\text { SIS (USA) = } \\
\text { PUHЦ (Russia) = } \\
\text { ESJI (KZ) = } \\
\text { SJIF (Morocco) }\end{array}$ & $\begin{array}{l}=0.912 \\
=0.179 \\
=1.042 \\
=\mathbf{2 . 0 3 1}\end{array}$ & ICV (Poland) & $=6.630$ \\
\hline
\end{tabular}

Решение задачи будем искать в виде произведения двух функций разных аргументов:

$$
U(x, t)=X(x) T(t) .
$$

Подставим (19) в уравнение (16), получим

$$
\begin{gathered}
X(x) T(t)\left(X^{I V}(x) T(t)+\alpha X^{\prime \prime}(x) T(t)\right)- \\
-\beta X(x) T^{\prime}(t)=0 \\
T^{2}(t)\left(X^{I V}(x)+\alpha X^{\prime \prime}(x)\right)=\beta T^{\prime}(t)=0,
\end{gathered}
$$

после разделения переменных будем иметь:

$$
X^{I V}(x)+\alpha X^{\prime \prime}(x)=\lambda ; \quad T^{\prime}(t)=\frac{\lambda}{\beta} T^{2}(t),
$$

Рассмотрим уравнение

$$
X^{I V}(x)+\alpha X^{\prime \prime}(x)=\lambda .
$$

Характеристическое уравнение, соответствующего однородного уравнения (21), будет иметь вид:

$$
\begin{array}{cl} 
& k^{4}+\alpha k^{2}=0, \\
k_{1,2}=0, & k_{3,4}= \pm \sqrt{-\alpha}= \pm i \sqrt{\alpha} .
\end{array}
$$

Следовательно, общее решение однородного уравнения примет вид:

$$
X_{\text {o.o }}=c_{1}+c_{2} x+c_{3} \cos \sqrt{\alpha} x+c_{4} \sin \sqrt{\alpha} x \text {. }
$$

Частное решение для уравнения (21) будем искать в виде:

$$
X_{\text {ч.H }}=A x^{2} e^{k x}=A x^{2} .
$$

Подставляя полученное в (21), находим

$$
A=\frac{\lambda}{2 \alpha}, \quad X_{\text {ч.H }}=\frac{\lambda}{2 \alpha} x^{2} .
$$

Следовательно, общее неоднородного уравнения будет иметь вид:

$$
\begin{aligned}
& X(x)=\frac{\lambda}{2 \alpha} x^{2}+c_{1}+c_{2} x+ \\
& +c_{3} \cos \sqrt{\alpha} x+c_{4} \sin \sqrt{\alpha} x .
\end{aligned}
$$

Удовлетворяя (22) условиям (17), получим систему уравнений для определения постоянных $c_{1}, c_{2}, c_{3}, c_{4}$ :

$$
\left\{\begin{array}{l}
c_{1}+c_{3}=0 \\
c_{2}+\sqrt{\alpha} c_{4}=0 \\
c_{1}+c_{2} l+c_{3} \cos \sqrt{\alpha} l+c_{4} \sin \sqrt{\alpha} l=-\frac{\lambda}{2 \alpha} l^{2} \\
c_{2}-\sqrt{\alpha} c_{3} \sin \sqrt{\alpha} l+\sqrt{\alpha} c_{4} \cos \sqrt{\alpha} l=-\frac{\lambda}{\alpha} l
\end{array}\right.
$$

Представим систему (23) в виде

$$
A C=B \text {, }
$$

где

$$
A=\left(\begin{array}{cccc}
1 & 0 & 1 & 0 \\
0 & 1 & 0 & \sqrt{\alpha} \\
1 & l & \cos \sqrt{\alpha} l & \sin \sqrt{\alpha} l \\
0 & 1 & -\sqrt{\alpha} \sin \sqrt{\alpha} l & \sqrt{\alpha} \cos \sqrt{\alpha} l
\end{array}\right),
$$

$$
C=\left(\begin{array}{l}
c_{1} \\
c_{2} \\
c_{3} \\
c_{4}
\end{array}\right), \quad B=\left(\begin{array}{c}
0 \\
-\frac{\lambda}{2 \alpha} l^{2} \\
-\frac{\lambda}{\alpha} l
\end{array}\right)
$$

Разрешая (23), находим

$$
\left\{\begin{aligned}
c_{1}= & \frac{\frac{\lambda}{2 \sqrt{\alpha}} l^{2}-\frac{\lambda}{\alpha} l \sin \sqrt{\alpha} l+\frac{\lambda}{2 \sqrt{\alpha}} l^{2} \cos \sqrt{\alpha} l}{2 \sqrt{\alpha}+2 \sqrt{\alpha} \cos \sqrt{\alpha} l-\alpha l \sin \sqrt{\alpha} l} \\
c_{2}= & \frac{\lambda}{\sqrt{\alpha}} l+\frac{\lambda}{\sqrt{\alpha}} l \cos \sqrt{\alpha} l+\frac{\lambda}{2} l^{2} \sin \sqrt{\alpha} l \\
& -\frac{\lambda}{2 \sqrt{\alpha}+2 \sqrt{\alpha} \cos \sqrt{\alpha} l-\alpha l \sin \sqrt{\alpha} l} l^{2} \cos \sqrt{\alpha} l-\frac{\lambda}{2 \sqrt{\alpha}} l^{2}+\frac{\lambda}{\alpha} l \sin \sqrt{\alpha} l \\
c_{3}= & \frac{\lambda \sqrt{\alpha}+2 \sqrt{\alpha} \cos \sqrt{\alpha} l-\alpha l \sin \sqrt{\alpha} l}{\lambda} l-\frac{\lambda}{\alpha} l \cos \sqrt{\alpha} l-\frac{\lambda}{2 \sqrt{\alpha}} l^{2} \sin \sqrt{\alpha} l \\
c_{4}= & \frac{\alpha \sqrt{\alpha}+2 \sqrt{\alpha} \cos \sqrt{\alpha} l-\alpha l \sin \sqrt{\alpha} l}{2 \sqrt{2} l}
\end{aligned}\right.
$$

Интегрируя второе из уравнений (20), получим

$$
T(t)=-\frac{\beta}{\lambda t+\beta c} .
$$

Принимая во внимание (19), (22), (24), при условии, что $\tau(x) \propto X(x)$, окончательно, будем иметь:

$$
\begin{gathered}
U(x, t)=-\frac{\beta}{\lambda t+\beta c}\left[\frac{\lambda}{2 \alpha} x^{2}+c_{1}+c_{2} x+\right. \\
\left.+c_{3} \cos \sqrt{\alpha} x+c_{4} \sin \sqrt{\alpha} x\right] .
\end{gathered}
$$

Таким образом (25) представляет собой решение задачи (16)-(18) и принадлежит требуемому классу функций.

\section{References:}

1. Ayshayev KM, Lesev VN (2007) To the theory of high-order nonlinear equations // Perspektiva-2007: Materials of the International Congress of Students, Post-graduate Students and Young Scientists. - Nalchik: KabardinoBalkarian University, 2007. - pp. 162-163.

2. Gegueva MM, Grinyuk VN, Kasumov UN, Lesev VN, Sozaev VA (2015) Thermodynamic 


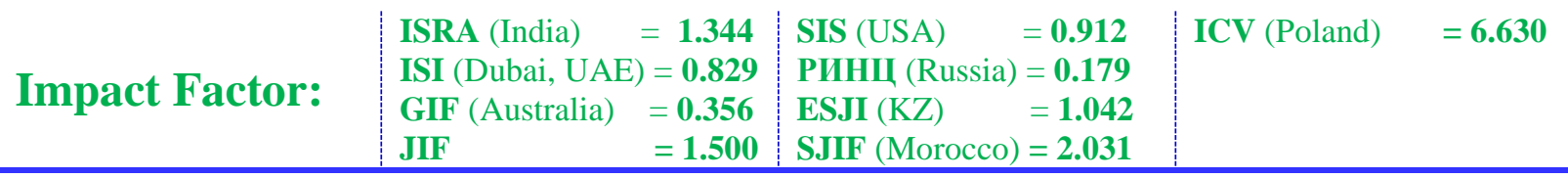

modelling of processes in zone-melting with electrical and thermal transfer in a liquid zone // News of the Russian Academy of Sciences. Physical Series. 2015. V. 79. No. 6. - pp. 814816.

3. Lesev VN (2012) The study of the mathematical model of capillary soaking in the magnetic field // Modern scientific research and their practical application. 2012. - Vol. J31209. - pp. 19-39.

4. Lesev VN, Sozaev VA (2010) Research of the problem about spreading of a liquid drop on a horizontal surface // News of Institutes of Higher Education. North-Caucasian Region. Natural sciences. 2010, No. 3. - pp. 28-31.

5. Lesev VN, Sozaev VA (2011) Research of small drops' statics and dynamics. Fundamental basics, mathematical models, numerical methods. - Saarbrücken (Germany): Lambert Academic Publishing. 2011. - pp.128.

6. Nahushev AM (1995) Equations of mathematical biology. - Moscow: Vysshaya shkola, 1995. - pp. 301.
7. Polyanin AD, Zhurov AI (2014) Functional constraints method for constructing exact solutions to delay reaction-diffusion equations and more complex nonlinear equations // Communications in Nonlinear Science and Numerical Simulation, 2014. Vol. 19, No. 3. pp. 417-430.

8. Polyanin AD, Zaytsev VF, Zhurov AI (2005) Methods of solving nonlinear equations of mathematical physics and mechanics. Moscow: Fizmatlit, 2005.

9. Nazarova LK (2014) Free boundary value problem for second-order quasi-linear equation // News of Smolensk State University. 2014. No. 3 (27). pp. 251-258.

10. Nazarova LK (2012) About one particular solution of a second-order partial differential equation with exponential nonlinearity // SouthSiberian Scientific Bulletin. 2012. No. 2. pp. 161-164. 